\title{
Adaptive Compressive Imaging for Object Reconstruction
}

\author{
Jun Ke, ${ }^{1,3 *}$ Amit Ashok, ${ }^{1}$ and Mark A. Neifeld, ${ }^{1,2}$ \\ ${ }^{1}$ Department of Electrical and Computer Engineering, University of Arizona, USA \\ ${ }^{2}$ College of Optical Science, University of Arizona, USA \\ ${ }^{3}$ Department of Electrical and Electronic Engineering, The University of Hong Kong, HK
}

\begin{abstract}
Static Feature-specific imaging (SFSI) employing a fixed/static measurement basis has been shown to achieve superior reconstruction performance to conventional imaging under certain conditions. ${ }^{1-5}$ In this paper, we describe an adaptive FSI system in which past measurements inform the choice of measurement basis for future measurements so as to maximize the reconstruction fidelity while employing the fewest measurements. An algorithm to implement an adaptive FSI system for principle component (PC) measurement basis is described. The resulting system is referred to as a PC-based adaptive FSI (AFSI) system. A simulation study employing the root mean squared error (RMSE) metric to quantify the reconstruction fidelity is used to analyze the performance of the PC-based AFSI system. We observe that the AFSI system achieves as much as 30\% lower RMSE compared to a SFSI system.
\end{abstract}

Keywords: Adaptive, Feature-specific imaging, Compressive sensing

\section{INTRODUCTION}

Feature-specific imaging $(\mathrm{FSI})^{1}$ is motivated by the fact that most natural scenes are inherently redundant and therefore, sparse in some transform domain such as Fourier and/or Wavelets. FSI employs transforms such as principal component (PC), Hadamard, Wavelets as the measurement basis. FSI imager directly measures linear projection(s) of the object on to the measurement basis and the resulting measurements are referred to as features. ${ }^{6}$ Typically, the number of measurements in a FSI system is much less than the object dimensionality, therefore, FSI is sometimes also referred to as compressive imaging in literature. ${ }^{7-11}$ Measuring fewer photon efficient measurements compared to traditional imaging, FSI can yield benefits such as improved measurement signal to noise ratio (SNR), reduced power, bandwidth, and weight requirements. Its superior performance to traditional imaging has been demonstrated for applications such as sensor network, structured illumination, and face detection. ${ }^{2-5}$

Previous research on FSI systems ${ }^{2,3,12}$ has utilized principal component or Karhunen-Loève basis for object reconstruction task. We refer to such a FSI system as a PC-based static FSI (SFSI) system, because the measurement bases are designed before the measurement process and remains fixed/static through the measurement process. Note that principle component (PC) basis incorporates the second-order correlation properties of object. For a SFSI system, we can design a PC basis that minimizes the reconstruction mean square error (MSE) for an ensemble of objects. However, such a basis is sub-optimal for any particular object. This forms the motivation to find a basis which incorporates individual object information as the measurement process progresses. Such a non-static measurement basis design would achieve a better reconstruction performance than a SFSI system. Here we take an adaptive approach that exploits past feature measurements to modify the measurement basis during measurement process. We refer to such a FSI system as an adaptive FSI (AFSI) system. As a PC basis is MSE optimal for object reconstruction assuming noise free measurements, ${ }^{13}$ here we use PC projection as an example to develop AFSI system and demonstrate its superior performance relative to SFSI system.

\footnotetext{
${ }^{*}$ Corresponding author: junke@email.arizona.edu, or junke@hku.hk
} 


\section{SFSI SYSTEM FRAMEWORK}

A SFSI system employs projection vectors drawn from a fixed measurement basis. Figure 1 shows a block diagram for a SFSI system. In this system, multiple images of an object are formed by a lenslet array. In the focal plane, each image of the object is modulated by a spatial light modulator (SLM) which displays one projection vector from the measurement basis. Light after the SLM is spatially integrated onto a single detector resulting in one feature measurement. An array of $L$ such projections yields $L$ feature measurements. After $I$ such measurements, $M=I L$ features are collected. An imager in figure 1 uses a parallel optical architecture,

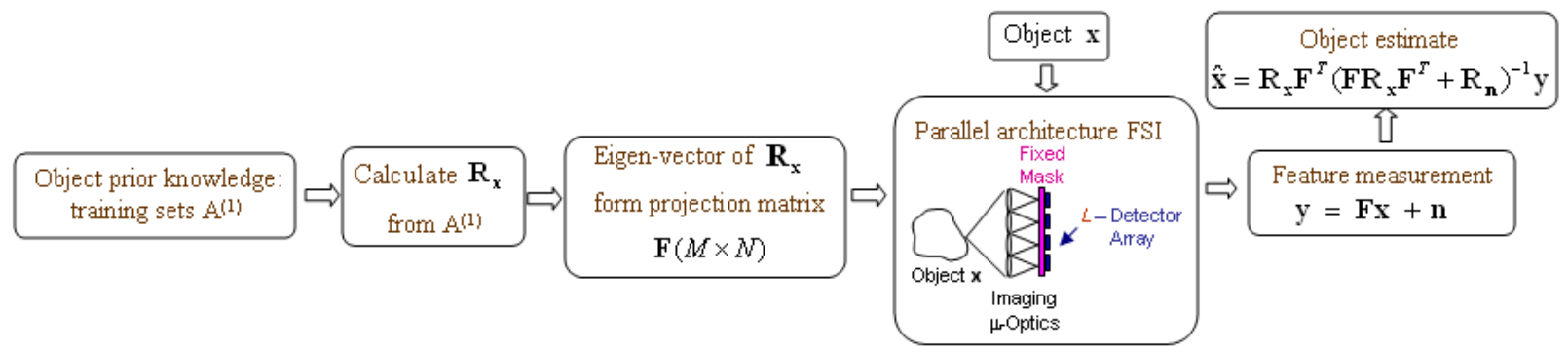

Figure 1. A block diagram for SFSI using PC bases.

because it collects $L$ feature measurements in parallel during one detector exposure time period $T_{0}$. In such a imager, the object irradiance collected in each feature measurement is reduced by a factor $L$ due to use of $L$ lenslets of equal diameter within the system aperture. ${ }^{12}$ The $M=I L$ feature measurements in a SFSI system can be represented as

$$
\mathbf{y}=\mathbf{F} \mathbf{x}+\mathbf{n}
$$

where $\mathbf{y}$ is the feature measurement vector of size $M \times 1, \mathbf{F}$ is the projection matrix of size $M \times N$ which includes the $1 / L$ factor resulting from choice of the parallel architecture, $\mathbf{x}$ is the object vector of size $N \times 1$ obtained by lexicographically arranging a $2 \mathrm{D}$ object of size $\sqrt{N} \times \sqrt{N}$, and $\mathbf{n}$ is the noise vector of size $M \times 1$. We assume the detector noise is additive white Gaussian noise with zero mean and variance is $\sigma^{2}=\sigma_{0}^{2} / T_{0}$. Here $\sigma_{0}^{2}$ is the noise energy per bandwidth, and $T_{0}$ is the detector exposure time. ${ }^{12}$ To reconstruct an object, we use a Wiener or a linear minimum mean square error (LMMSE) operator,

$$
\mathbf{W}=\mathbf{R}_{x} \mathbf{F}^{T}\left[\mathbf{F} \mathbf{R}_{x} \mathbf{F}^{T}+\mathbf{R}_{n}\right]^{-1}
$$

where $\mathbf{R}_{x}$ and $\mathbf{R}_{n}$ are the object and noise auto-correlation matrices respectively, $\mathbf{R}_{n}=\sigma^{2} \mathbf{I}$. The estimated object $\hat{\mathbf{x}}$ is $\hat{\mathbf{x}}=\mathbf{W y}$.

The projection matrix $\mathbf{F}$ in a PC-based SFSI (PC-SFSI) system is constructed from eigenvectors of the object auto-correlation matrix $\mathbf{R}_{x}$. To minimize reconstruction error $M$ eigenvectors corresponding to the largest $M$ eigenvalues are used to form the PC projection matrix $\mathbf{F}$, yielding $\mathrm{M}$ features. ${ }^{13}$ With this projection matrix $\mathbf{F}$, the reconstruction MSE is minimized for an ensemble of objects when they satisfy an underlying Gaussian distribution. ${ }^{13}$ However, for a specific object, this PC projection matrix is sub-optimal and therefore the projection vectors need to be designed specifically for that object. For example, we observe a set of $M$ PC projection vectors derived from a training set comprised of "tank" or "face" yield a smaller reconstruction MSE values for a "tank" or a "face" object respectively, compared with a set of $M$ PC projection vectors derived from a larger training set spanning a wider class of objects such as building, mountain, or river. Note that the first set of projection vectors are derived from a set composed of training samples similar to the test objects. Therefore, the projection vectors are able to incorporate more relevant object prior knowledge compared to a set composed of different class of data samples than the test object. This observation motivates our design for a PC-based AFSI system discussed in the next section.

\section{AFSI SYSTEM FRAMEWORK}

In an PC-based AFSI system, the feature measurement basis is modified based on the set of past PC feature measurements. For example, for a $\sqrt{N} \times \sqrt{N}$ object, the first $L$ PC projection vectors are derived from a 
training set, then they are used to make the corresponding $L$ feature measurements. Based on these $L$ PC feature measurements, the second $L$ projections are derived and the second $L$ feature measurements are collected and so on. Following this procedure, $M$ PC projection vectors are designed sequentially incorporating object knowledge learnt at each measurement step.

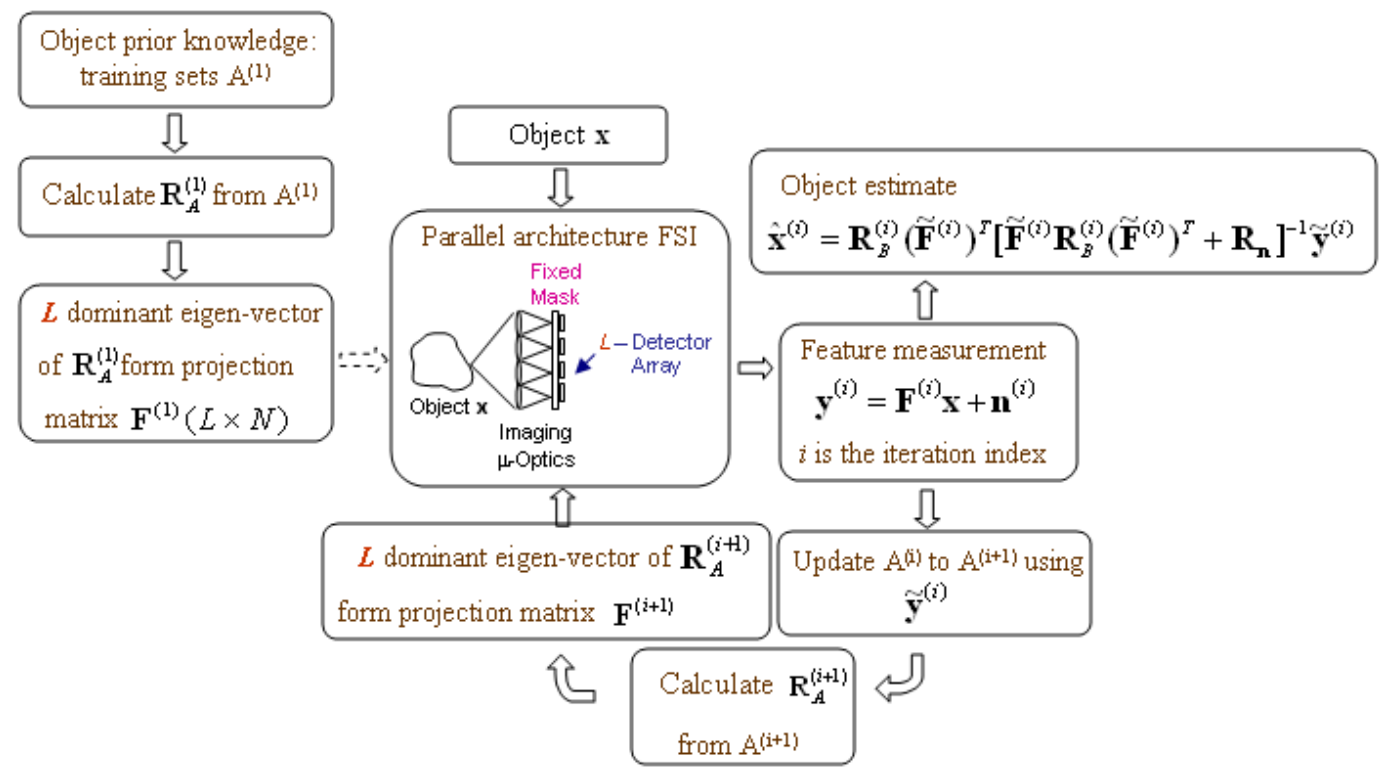

Figure 2. A block diagram for AFSI using a PC basis

Figure 2 presents the block diagram of a PC-AFSI system. Before continuing the discussion, we define a list of parameters as shown below:

$\mathbf{x}$ : object vector of size $N \times 1$.

$A^{(i)}, B^{(i)}$ : two training sets in the $i^{\text {th }}$ iteration, where samples in $A^{(i)}$ span a $N-i L$ dimensional space and samples in $B^{(i)}$ span a $N$ dimensional space.

$k(i)$ : cardinality of $A^{(i)}$ and $B^{(i)}$.

$\mathbf{R}_{A}^{(i)}, \mathbf{R}_{B}^{(i)}$ : auto-correlation matrices estimated from samples in $A^{(i)}$ and $B^{(i)}$.

$\mathbf{F}^{(i)}$ : projection matrix of size $L \times N$ in the $i^{\text {th }}$ iteration.

$\widetilde{\mathbf{F}}^{(i)}$ : accumulated projection matrix of size $(i L) \times N$ after the first $i$ iterations.

$\mathbf{n}^{(i)}$ : noise vector of size $L \times 1$ in the $i^{\text {th }}$ iteration.

$\mathbf{y}^{(i)}$ : feature vector of size $L \times 1$ in the $i^{\text {th }}$ iteration.

$\widetilde{\mathbf{y}}^{(i)}$ : accumulated feature vector of size $(i L) \times 1$ after the first $i$ iterations.

$\mathbf{W}_{n}^{(i)}$ : reconstruction operator in the $i^{\text {th }}$ iteration using measurement with noise.

$\hat{\mathbf{x}}^{(i)}$ : object reconstruction in the $i^{\text {th }}$ iteration.

As shown in figure 2, $L$ PC projection vectors in each adaptation iteration are derived from a training set $A^{(i)}$. These $\mathrm{PC}$ vectors are the $L$ dominant eigenvectors of $\mathbf{R}_{A}^{(i)}$, where $i$ denotes the $i^{\text {th }}$ iteration, $i=1,2,3, \ldots, I$, and $I$ is the total number of adaptation steps. Feature measurements $\mathbf{y}^{(i)}$ in the $i^{\text {th }}$ iteration are represented as

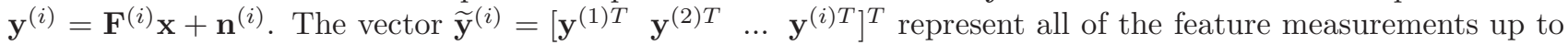
the $i^{\text {th }}$ iteration. At each iteration, additional information about the object is incorporated by computing new $\mathrm{PC}$ projection vectors from an updated training set that is in turn defined by last training set and the feature 
measurements acquired until that step. The adaptation process employs denoised feature measurements, which are defined as

$$
\hat{\mathbf{y}}^{(i)}=\mathbf{W}_{y}^{(i)} \mathbf{y}^{(i)}
$$

where $\mathbf{W}_{y}^{(i)}=\mathbf{R}_{y}^{(i)}\left[\mathbf{R}_{y}^{(i)}+\mathbf{R}_{n}\right]^{-1}$ and $\mathbf{R}_{y}^{(i)}=E\left\{\left[\mathbf{F}^{(i)} \mathbf{x}\right]\left[\mathbf{F}^{(i)} \mathbf{x}\right]^{T}\right\}=\mathbf{F}^{(i)} \mathbf{R}_{B}^{(i)} \mathbf{F}^{(i) T}$. The " $K$-nearest neighbor" method is used to update training set $A^{(i)}$ to $A^{(i+1)}$ in the accumulated feature space. In this method, we choose the $k(i+1)$ closest training samples to $\hat{\mathbf{x}}^{(i)}$ from $A^{(i)}$. These chosen samples define the updated training set $A^{(i+1)}$ and the remaining samples are discarded. We also remove the subspace spanned by the $i L$ PC projections from $A^{(i+1)}$ to ensure orthogonality among all projection vectors. The number of training samples $k(i)$ at $i^{\text {th }}$ iteration is defined as: $k(i)=\alpha e^{-\beta i}$, the the training set size decreases exponentially as $i$ increases. Parameters $\alpha$ and $\beta$ are optimized based on more than 50 different training sets. The optimal values of $\alpha=0.5$ and $\beta=0.0063$ maximizes AFSI system performance and are used throughout the remainder of this paper.

To estimate an object from its $i L$ PC feature measurements, we use the Wiener/LMMSE operator. The object estimate is $\hat{\mathbf{x}}^{(i)}=\mathbf{W}_{n}^{(i)} \widetilde{\mathbf{y}}^{(i)}$, where $\mathbf{W}_{n}^{(i)}$ is the Wiener/LMMSE operator $\mathbf{W}_{n}^{(i)}=\mathbf{R}_{B}^{(i)} \widetilde{\mathbf{F}}^{(i) T}\left[\widetilde{\mathbf{F}}^{(i)} \mathbf{R}_{B}^{(i)} \widetilde{\mathbf{F}}^{(i) T}+\mathbf{R}_{n}\right]^{-1}$. In the mathematical formulation for $\mathbf{W}_{n}^{(i)}, \widetilde{\mathbf{F}}^{(i)}=\left[\begin{array}{llll}\mathbf{F}^{(1) T} & \mathbf{F}^{(2) T} & \cdots & \mathbf{F}^{(i) T}\end{array}\right]^{T}$ is the accumulated projection matrix and $\mathbf{R}_{n}=\sigma^{2} \mathbf{I}$ is the noise autocorrelation matrix. Note that, $\mathbf{R}_{B}^{(i)}$ is derived from the sample set $B^{(i)}$ with $B^{(1)}=A^{(1)}$ initially. $B^{(i)}$ is also updated using the same " $K$-nearest neighbor" method as used for updating $A^{(i)}$. But the subspace spanned by the $i L$ projections is not removed from $B^{(i+1)}$ in order to make $\mathbf{R}_{B}^{(i)}$ an autocorrelation matrix for objects in a $N$ dimensional space instead of a $N-i L$ dimensional space.

The algorithm for estimating the object $\mathbf{x}$ using $I$ iterations in a PC-AFSI system is summarized as following:

1. Calculate $\mathbf{R}_{A}^{(i)}=E\left\{\mathbf{a}_{j}^{(i)} \mathbf{a}_{j}^{(i) T}\right\}$ and $\mathbf{R}_{B}^{(i)}=E\left\{\mathbf{b}_{j}^{(i)} \mathbf{b}_{j}^{(i) T}\right\}$, where $E\{\}$ represents the mathematical expectation, $\mathbf{a}_{j}^{(i)}$ and $\mathbf{b}_{j}^{(i)}$ are training samples in $A^{(i)}$ and $B^{(i)}$ respectively, $j=1,2, \cdots, k(i)$. Initially, $A^{(1)}=B^{(1)}$ and $k(1)=\alpha e^{-\beta}=0.5 e^{-0.0063}$.

2. Calculate the $L$ dominant eigen-vectors of $\mathbf{R}_{A}^{(i)}$ and form $\mathbf{F}^{(i)}$.

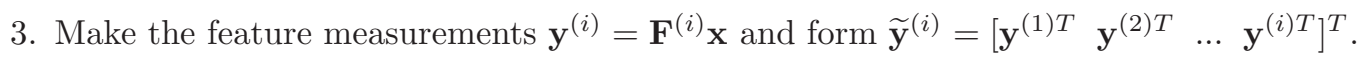

4. Estimate the object $\hat{\mathbf{x}}^{(i)}=\mathbf{W}^{(i)} \widetilde{\mathbf{y}}^{(i)}$

5. Calculate the de-noised feature vector $\hat{\mathbf{y}}^{(i)}=\mathbf{W}_{y}^{(i)} \mathbf{y}^{(i)}$.

6. Calculate the feature measurements of $\mathbf{a}_{j}^{(i)}, \mathbf{u}_{j}^{(i)}=\mathbf{F}^{(i)} \mathbf{a}_{j}^{(i)}$. Form $\widetilde{\mathbf{u}}_{j}^{(i)}=\left[\begin{array}{llll}\mathbf{u}_{j}^{(1) T} & \mathbf{u}_{j}^{(2) T} & \cdots & \mathbf{u}_{j}^{(i) T}\end{array}\right]^{T}$.

7. Calculate the distance between $\hat{\mathbf{x}}^{(i)}$ and $\mathbf{a}_{j}^{(i)}$ in feature space: $d_{j}^{(i)}=\left\|\widetilde{\mathbf{u}}_{j}^{(i)}-\widetilde{\hat{\mathbf{y}}}^{(i)}\right\|_{2}$, where $\tilde{\hat{\mathbf{y}}}^{(i)}=\left[\begin{array}{llll}\hat{\mathbf{y}}^{(1) T} & \hat{\mathbf{y}}^{(2) T} & \ldots & \hat{\mathbf{y}}^{(i) T}\end{array}\right]^{T}$.

8. Update $A^{(i)}$ to $A^{(i+1)}$ by choosing $\mathbf{a}_{j}^{(i)}$ such that $\mathbf{a}_{j}^{(i)}$ is associated with the $k(i+1)$ smallest $d_{j}^{(i)}$ values. $B^{(i)}$ is also updated to $B^{(i+1)}$ accordingly.

9. Define the samples in $A^{(i+1)}$ as $\mathbf{a}_{j}^{(i+1)}=\mathbf{a}_{j}^{(i)}-\mathbf{F}^{(i) T} \mathbf{u}_{j}^{(i)}$.

10. If $i<I$, go back to 1 .

\section{SIMULATION RESULTS}

In the simulation study of PC-based SFSI and AFSI systems, we use 97,392 image samples of size $32 \times 32$, of object such as face, building, and tank images to form the initial training set $A^{(1)}$. These samples with dynamic range of $[0,255]$ are obtained under high, moderate and low illumination conditions. Six such training samples are presented in the top row of figure 3. Twenty tank object images are used for testing. Detector noise energy per 
bandwidth in the FSI systems is set to $\sigma_{0}^{2}=1$ throughout the rest of this section. With detector exposure time $T_{0}$ the noise variance in feature measurement is $\sigma^{2}=\sigma_{0}^{2} / T_{0}=1 / T_{0}$. In figure 3 , first row shows the original samples, the second row presents the noisy measurements of the training samples acquired using a conventional imager where $T_{0}=0.02 \mathrm{~s}$. Reconstruction error is quantified with the relative RMSE metric, $E\left\{\left\|\hat{\mathbf{x}}^{(i)}-\mathbf{x}\right\|_{2} /\|\mathbf{x}\|_{2}\right\}$, where $E\{\}$ denotes the mathematical expectation over the testing set. To simplify notation, we also use RMSE here onwards to refer to relative RMSE.
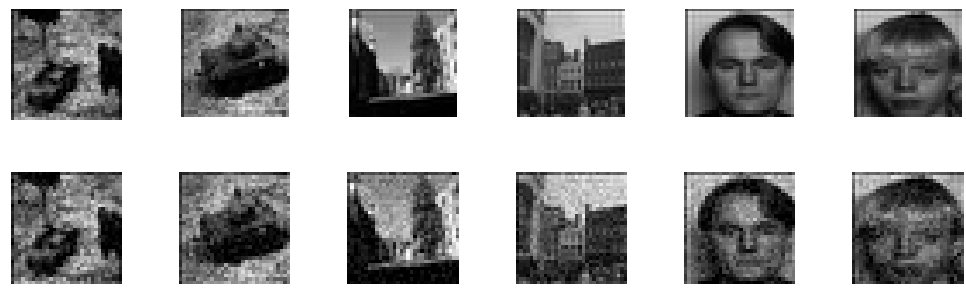

Figure 3. Some object examples (top row) and its noisy measurements when $\sigma^{2}=1 / T_{0}$ with $T_{0}=0.02 s$ (bottom row).

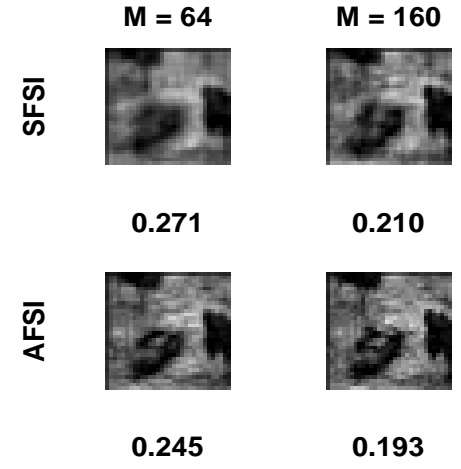

(a)

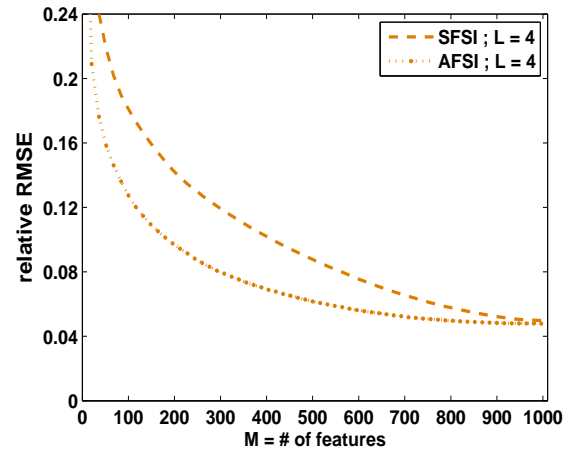

(b)

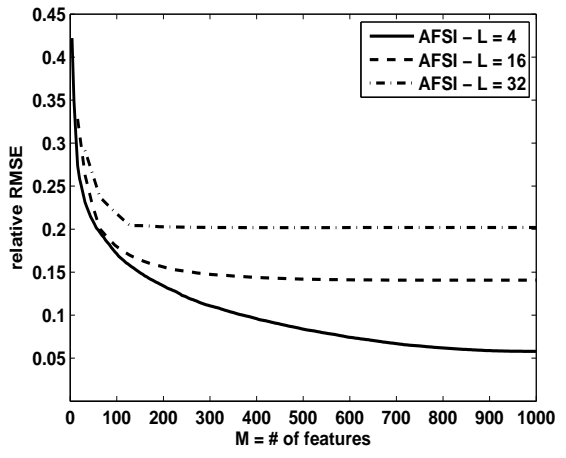

(c)

Figure 4. a) reconstruction examples; RMSE vs. M using b) PC AFSI and SFSI for $L=4$ when $T_{0}=0.02 s$; c) PC AFSI for different $L$.

Four reconstruction examples of an object using SFSI and AFSI systems are presented in the top and bottom rows in figure $4 \mathrm{a}$ ), respectively. The original object is the left most sample in the top row of figure 3 . For both of the SFSI and AFSI systems the corresponding object estimates, obtained from $M=64$ and 160 feature measurements, are shown in the left and right columns in figure 4 a), respectively. The reconstruction error RMSE value each object is labeled under the corresponding object estimate. From figure 4 a), we observe that PC-based AFSI shows both visually and quantitatively superior reconstruction performance compared to PC-based SFSI. The RMSE values for the reconstructions in AFSI when $M=64$ and $M=160$ are 0.245 and 0.193 respectively, compared with the corresponding RMSE values of 0.271 and 0.210 for the reconstructions in SFSI system. Visually the reconstructions in AFSI present better quality than the reconstructions in SFSI with more contrast and details. Figure $4 \mathrm{~b}$ ) presents a plot of average reconstruction error RMSE vs. $M$ for PC-based SFSI and AFSI systems using 20 tank testing samples with $L=4$ and $T_{0}=0.02 \mathrm{~s}$. We can make the following observations from this figure. First, for both systems the reconstruction error reduces and approaches towards a minimum value as $M$ increases. The non-zero minimum RMSE value as $M=N$ is due to a finite measurement SNR. Two components contribute to the reconstruction error: the truncation error and the feature measurement SNR. The truncation error results from using $M<N$ feature measurements. Therefore, it reduces as $M$ increases towards $N$ as high order features are used towards the reconstruction. However, note that the feature measurement SNR 
decreases as $M$ increases, because a smaller object irradiance is available per feature under constant exposure and fixed detector noise constraints. The LMMSE reconstruction operator suppresses the contribution of a feature to the object estimate if its measurement SNR is too small. Therefore, the feature measurement SNR ultimately limits the achievable minimum RMSE and the $M$ value at which it is first achieved. The second observation from figure $4 \mathrm{~b}$ ) is that the PC-base AFSI system yields a superior reconstruction performance relative to the PC-based SFSI system in terms of the RMSE metric. For example, when $M=260$, the RMSE for AFSI is 0.086 which is $32.3 \%$ less than the RMSE of 0.127 for SFSI. In figure $4 \mathrm{c}$ ) a plot of RMSE vs. $M$ for the PC-based AFSI system with $L=4,16$, and 32 is shown. Note that, with increasing $L$ the reconstruction RMSE increases due to reduced feature measurement SNR for every object feature. This can be understood by noting that in a parallel architecture FSI system with $L$ measurements, the signal energy in each feature measurement reduces as $L$ increases, while the detector noise variance is fixed as $1 / T_{0}$. Therefore, the system reconstruction performance reduces as a result of decreasing feature measurement SNR with increasing $L$. Another observation from figure $4 \mathrm{c}$ ) is that the minimum reconstruction RMSE achieved is reached first at a lower M as $L$ increases. This is due to the fact that the Wiener reconstruction operator effectively limits the use of feature measurement with low SNR to minimize reconstruction error. Thus as $L$ increases and each feature measurement SNR decreases with increasing $L$, the FSI system achieves its minimum error with fewer feature measurements.

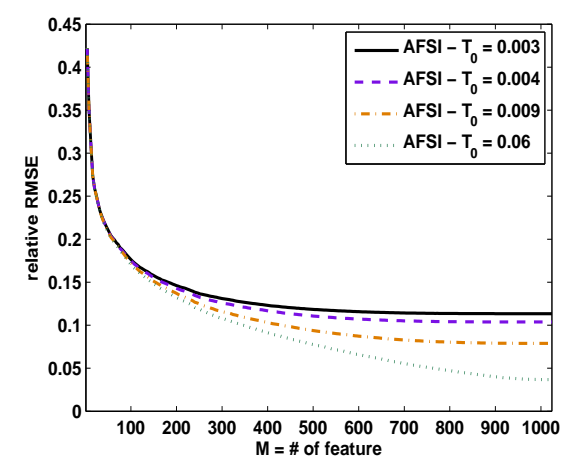

Figure 5. RMSE vs. $M$ for different $T_{0}(s)$ in PC-based AFSI system when $L=32$.

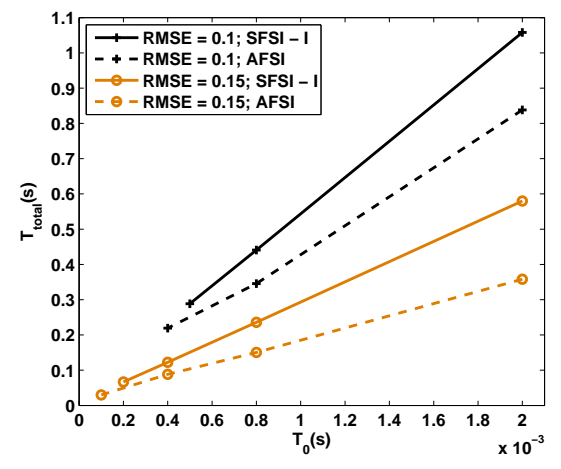

(a)

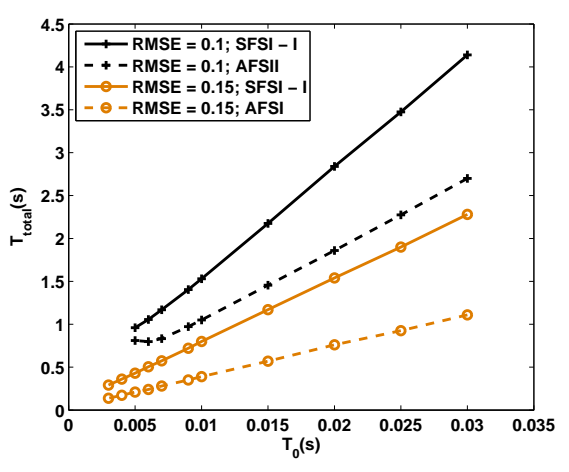

(b)

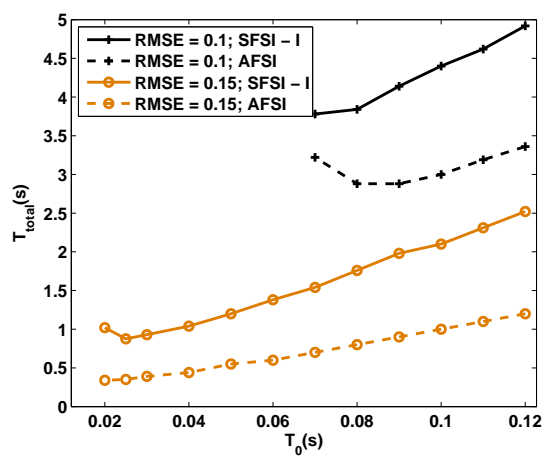

(c)

Figure 6. $T_{\text {total }}$ vs. $T_{0}$ for a) $L=1$, b) $L=4$, c) $L=16$ PC-based SFSI and AFSI systems.

Figure 5 shows the reconstruction RMSE in the PC-based AFSI varies as a function of $M$ for different detector exposure time $T_{0}$ when $L=32$. As expected, the AFSI system has smaller reconstruction error for larger $T_{0}$, because the noise energy is inversely proportional to $T_{0}$ hence low detector noise and large measurement SNR 
results from a large $T_{0}$ value. Given a RMSE value, we can find the minimum number of features $M_{\min }$ and the total feature collection time $T_{\text {total }}=T_{0} * M_{\min }$ required to reach a desired reconstruction error. Figures 6 a), b), and c) show $T_{\text {total }}$ vs. $T_{0}$ in PC-based SFSI and AFSI systems respectively with $L=1,4,16$. The desired RMSE values are set to 0.10 and 0.15 . $T_{\text {total }}$ value is determined by two underlying mechanisms. For large $T_{0}$ value, the measurement noise is small, but it requires more time to acquire the data achieving the desired RMSE value hence the system loses its adaptation advantage. For small $T_{0}$ value, although the measurement noise is large, the system is more agile and can more quickly exploit knowledge acquired from measurements. Therefore, for each $L$ there exists a minimum $T_{\text {total }}$ at which the measurement noise and adaptation balance out each other. Several such minimum $T_{\text {total }}$ values are presented in figures $6 \mathrm{~b}$ ) and c). Note that, if $T_{0}$ is too small or the detector noise is too large, FSI system can not reach small desired RMSE value, therefore, some curves in figures 6 have their minimum $T_{\text {total }}$ values at the smallest $T_{0}$ values. The second observation is that $T_{\text {total }}$ in an AFSI system is always smaller than the $T_{\text {total }}$ values in a SFSI system as expected. For example, with $L=16$ the minimum $T_{\text {total }}$ to achieve RMSE of 0.15 in PC-based AFSI system is $0.34 s$, which is 2.57 times less than the minimum $T_{\text {total }}$ value $0.875 \mathrm{~s}$ in PC-based SFSI system. The minimum $T_{\text {total }}$ values for both PC-based AFSI and SFSI systems for $L=1,4$ and 16 are listed in tables 1 . From figure 6 and table 1 , we also have the observation that the minimum $T_{\text {total }}$ values for FSI systems increases as $L$ increases due to the reduced measurement SNR for increasing $L$. From this observation we can conclude when there is finite measurement noise, the AFSI system with $L=1$ presents the best performance among all the parallel architecture AFSI systems.

Table 1. minimum $T_{\text {total }}$ in PC-based SFSI and AFSI systems for $L=1,4$, and 16

\begin{tabular}{cccccc}
\hline \multirow{2}{*}{$L$} & \multirow{2}{*}{ RMSE } & \multicolumn{2}{c}{ SFSI } & \multicolumn{2}{c}{ AFSI } \\
\cline { 2 - 6 } & & $T_{0}(\mathrm{~ms})$ & $T_{\text {total }}(\mathrm{s})$ & $T_{0}(\mathrm{~ms})$ & $T_{\text {total }}(\mathrm{s})$ \\
\hline \multirow{2}{*}{1} & 0.10 & 0.4 & 0.2884 & 0.4 & 0.2192 \\
\cline { 2 - 6 } & 0.15 & 0.1 & 0.0668 & 0.1 & 0.03 \\
\hline \multirow{2}{*}{4} & 0.10 & 5 & 0.96 & 6 & 0.798 \\
\cline { 2 - 6 } & 0.15 & 3 & 0.291 & 3 & 0.138 \\
\hline \multirow{2}{*}{16} & 0.10 & 70 & 3.78 & 80 & 2.88 \\
\cline { 2 - 6 } & 0.15 & 25 & 0.875 & 20 & 0.34 \\
\hline
\end{tabular}

\section{CONCLUSION}

In this paper, we analyzed an adaptive FSI system for reconstruction task using PC projections. A training set including more than 97,000 samples is used to design the PC projections. The adaptation is achieved by updating the training set based on the collected feature measurements. We also developed an algorithm for the PC-based AFSI systems using noisy measurements. The superior reconstruction performance of AFSI relative to SFSI is demonstrated using parallel architecture FSI systems for $L$ feature measurements. The improvement of reconstruction RMSE from PC-based SFSI system to PC-based AFSI system is about $30 \%$ for tank testing samples. The AFSI system also required smaller exposure time $T_{\text {total }}$ to achieve a desired reconstruction RMSE value compared with the SFSI system. It is observed that the AFSI system with $L=1$ presents the best system performance.

\section{ACKNOWLEDGMENTS}

The authors want to acknowledge Dr. Lam, the Imaging Systems Laboratory, and the Department of Electrical and Electronic Engineering of the University of Hong Kong for their support to the publication of this paper.

\section{REFERENCES}

[1] Neifeld, M. A. and Shankar, P. M., "Feature specific imaging," Applied Optics 42, 3379-3389 (June 2003).

[2] Ke, J., Shankar, P. M., and Neifeld, M. A., "Distributed imaging using an array of compressive cameras," Optics Communications 282, 185-197 (2009).

[3] Baheti, P. K. and Neifeld, M. A., "Feature-specific structured imaging," Applied Optics 45, 7382-7391 (Oct 2006). 
[4] Pitsianis, N., Brady, D., Portnoy, A., Sun, X., Suleski, T., Fiddy, M., Feldman, M., and TeKolste, R., "Compressive imaging sensors," in [Proc. of SPIE Vol], 6232, 62320A-1.

[5] Pitsianis, N., Brady, D., and Sun, X., "Sensor-layer image compression based on the quantized cosine transform," in [Proc. of SPIE Vol], 5817, 251.

[6] Agaian, S., [Hadamard Matrices and Their Applications], Springer-Verlag (1985).

[7] Tsaig, Y. and Donoho, D., "Extensions of compressed sensing," Signal Processing 86, 549-571 (March 2006).

[8] Cand'es, E. J. and Wakin, M. B., "People hearing without listening:an introduction to compressive sampling," IEEE Signal Processing Magazine (to appear).

[9] Jarvis Haupt, R. N., "Signal reconstruction from noisy random projections," IEEE Trans. on Information Theory 52, 4036-4048 (Sep 2006).

[10] Takhar, D., Laska, J., Wakin, M., Duarte, M., Baron, D., Sarvotham, S., Kelly, K., and Baraniuk, R., "A new compressive imaging camera architecture using optical-domain compression," ISEST/SPIE Computational Imaging IV (2006).

[11] Duarte, M., Davenport, M., Takhar, D., Laska, J., Sun, T., Kelly, K., and Baraniuk, R., "Single-pixel imaging via compressive sampling," IEEE Signal Processing Magazine 25(2), 83-91 (2008).

[12] Ke, J. and Neifeld, M. A., "Optical architectures for compressive imaging," Applied Optics 46, 5293-5303 (Aug 2007).

[13] Jolliffe, I., [Principle Component Analysis], Springer (2002). 